\title{
Estructura e invarianza factorial de una escala de perfeccionismo infantil adaptada para adolescentes
}

\author{
Cristian Ramos-Vera ${ }^{1,2}$, Annie Dávila-Campos ${ }^{1}$, Jeack Castañeda-Barros ${ }^{1}$, Yelitza Ramírez-Ramírez ${ }^{1}$ \\ y Antonio Serpa-Barrientos ${ }^{1,2,3}$ \\ ${ }^{1}$ Universidad Cesar Vallejo, Lima, Perú \\ ${ }^{2}$ Sociedad Peruana de Psicometría, Lima, Perú \\ ${ }^{3}$ Universidad Nacional Mayor de San Marcos, Lima, Perú
}

Structure and factorial invariance of a child perfectionism scale adapted for adolescents

\begin{abstract}
The aim of the present study was to obtain the psychometric properties of the Child perfectionism scale (Oros, 2003) adapted for adolescents, in 917 Peruvian adolescents, aged 13 to 18 years $(M=15.241, D T=1.020)$. It was analyzed through confirmatory factor analysis, showing that the three-factor model had best parsimony with optimal fit indices $\left(\mathrm{S}-\mathrm{B} \chi^{2} / \mathrm{gl}=0.51\right.$; $\mathrm{CFI}=.95, \mathrm{SRMR}=.056$, RMSEA [IC 90\%] $=.055$ [.049-.061]). Measurement invariance across sex was also evaluated. There was evidence of reliability and evidence of convergent validity regarding the Reynold Adolescent Depression Scale by means of Pearson's correlation and network analysis.
\end{abstract}

Keywords: Perfectionism; depression; factorial invariance; adolescents; network analysis.

Resumen: El objetivo de la investigación consistió en obtener las propiedades psicométricas de la adaptación para adolescentes de la escala de perfeccionismo infantil de Oros (2003) en 917 adolescentes peruanos, comprendido entre 13 y 18 años $(M=15.241$, $D T=1.020)$. Mediante análisis factorial confirmatorio, se evidenció mejor parsimonia en el modelo de tres factores con óptimos índices de ajuste $\left(\mathrm{S}-\mathrm{B} \chi^{2} / \mathrm{gl}=0.51 ; \mathrm{CFI}=.95, \mathrm{SRMR}=.056\right.$, RMSEA [IC 90\%] $=.055$ [.049-.061]). También se evaluó equivalencia de medida según sexo. Se proporciona evidencia de fiabilidad y validez convergente con la medida del Reynold Adolescent Depression Scale mediante la correlación de Pearson y análisis de red.

Palabras clave: Perfeccionismo; depresión; invarianza factorial; adolescentes; análisis de red.

\section{Introducción}

Se estima que, entre dos cuartos y dos tercios de la población, independientemente de la edad, podrían presentar rasgos negativos perfeccionistas (Kelly y Zuroff, 2013), estas personas reportan altos niveles de esfuerzos perfeccionistas y altos niveles de preocupaciones por un rendimiento perfecto (Stoeber y Childs, 2012).

Recibido: 25 de febrero 2020; aceptado: 22 de abril 2021

Correspondencia: Cristian Ramos Vera, Facultad de Ciencias de la Salud, Universidad Cesar Vallejo, Av. Del Parque 640, San Juan de Lurigancho 15434, Perú. Correo-e: cristony_777@hotmail.com
Actualmente los estudios sobre el perfeccionismo mayormente abarcan a la población adulta y universitaria (Morris y Lomax, 2014). Las investigaciones del perfeccionismo durante la infancia y la adolescencia representan un campo relativamente nuevo (Hewitt et al., 2002, Morris y Lomax, 2014), en parte debido a la escasez de instrumentos específicos para estos grupos etarios (Leone y Wade, 2017). Aunque últimamente se han propuesto varios instrumentos como la Escala de perfeccionismo multidimensional de Frost (Frost et al., 1990), la escala de perfeccionismo infantil-adolescente (Flett et al., 2000), la escala de perfeccionismo multidimensional (Hewitt y Flett, 1991) y la escala de perfeccionismo infantil (EPI) de Oros (2003) que han permitido el crecimiento de las in- 
vestigaciones florecientes en el área del perfeccionismo infantojuvenil (García-Fernández et al., 2016).

El perfeccionismo es una disposición de la personalidad caracterizada por la búsqueda de la perfección y la fijación de normas de rendimiento excesivamente elevadas, acompañada de evaluaciones muy autocríticas (Frost et al., 1990). Varios estudios han indicado al perfeccionismo como un concepto multidimensional compuesta por varias facetas perfeccionistas como el perfeccionismo orientado al esfuerzo y el perfeccionismo autocrítico (p.ej., Frost et al., 1993; Stoeber y Otto, 2006).

La faceta del perfeccionismo orientado al esfuerzo que comprende los estándares personales elevados y un empeño propio por la perfección (Stoeber y Childs, 2012). Mientras, el dominio vinculado al perfeccionismo autocrítico incluye las preocupaciones perfeccionistas caracterizadas por un malestar ante los errores cometidos y las reacciones negativas autocriticas (Frost et al., 1993; Stoeber y Otto, 2006). Siendo dos dimensiones complementarias de la personalidad perfeccionista (Stoeber, 2018) que son evaluadas por varias medidas del perfeccionismo incluyendo la EPI de Oros (2003), este instrumento está compuesto por el factor de autodemandas (perfeccionismo orientado al esfuerzo) y las reacciones frente al fracaso (perfeccionismo autocrítico).

La etapa de adolescencia es un periodo importante y de transición que puede conllevar a una mayor predominancia del perfeccionismo (Aguilar y Castellanos, 2017), siendo un posible factor de riesgo de problemas a nivel psicológico (Flett et al., 2016). En particular, en el ámbito escolar destacan comportamientos perfeccionistas de los adolescentes como la meticulosidad en el estudio, una preocupación excesiva por obtener altos niveles de desempeño acompañado de una preocupación por no fallar en las áreas académicas (Rimm, 2007). Otra investigación reporta que tres de cada diez adolescentes tienden a ser perfeccionistas y los demás presentan algunos rasgos de perfeccionismo (Sironic y Reeve, 2015).

Diversas investigaciones manifiestan que el perfeccionismo está vinculado a varios trastornos psicopatológicos (Limburg et al., 2017) como: insatisfacción corporal (Nigar y Naqvi, 2019), ansiedad y depresión (Hewitt et al., 2002), ideación suicida (Flett et al., 2016), anorexia y trastorno obsesivo compulsivo (Flamarique et al., 2019), burnout (Hill y Curran, 2016), rumia psicológica (Flett et al., 2011) y diversos pensamientos irracionales o errores cognitivos (Davis y Wosinski, 2012). Además, el perfeccionismo actuaría como un factor de resistencia al cambio ante los tratamientos clínicos de la salud mental (Lloyd et al., 2015; Van der Kaap-Deeder et al., 2016).

El instrumento que se utilizó para el presente estudio fue la escala de perfeccionismo infantil (EPI) de Oros
(2003), en la investigación original de la EPI se evaluó a una muestra de 583 niños pertenecientes a las ciudades de Buenos Aires y Entre Ríos (Argentina), mediante el análisis factorial exploratorio se realizaron el proceso de construcción de la prueba, obteniendo dos factores: autodemandas y reacciones frente al fracaso (Oros, 2003).

La EPI también fue estudiada en el contexto venezolano, por Aguilar y Castellanos (2017) en una muestra de 342 estudiantes entre las edades de 11 y 15 años, teniendo cuatro dimensiones: autodemandas, culpa y remordimiento, malestar, y autocastigo. Se realizó el análisis de componentes principales y el método de rotación ortogonal varimax, obteniendo el $50.2 \%$ de la varianza total explicada, el método usado es cuestionable cuando se tiene el fin de explorar la dimensionalidad del contructo, cuya medida de confiabilidad general de alfa de Cronbach fue de .76 .

Finalmente Ventura et al. (2018) realizaron una validación de la Escala del Perfeccionismo en una muestra de 1084 niños peruanos entre 7 a 12 años, tomando en cuenta las dos dimensiones de la versión original, presentando aceptables niveles de validez $(\mathrm{KMO}=.73 \mathrm{y}$ prueba de esfericidad de Bartlett $=620.4, p<.001$ ), ambos componentes explican el $44 \%$ de la varianza total del constructo, y solo presentaron la confiabilidad general de coeficiente de omega $(\omega=.83)$ y no por dimensiones. Este estudio incluyó el análisis factorial confirmatorio, evaluando tres modelos: primero la versión original, y un segundo modelo que eliminó los ítems 4,10 y 12 , y el tercer modelo basado en el anterior de dos factores oblicuos de 13 ítems con errores correlacionados entre items 2-8, 9-11 y 14-16, mostrando mejores bondades de ajustes $\left(\chi^{2} / \mathrm{gl}=2.21\right.$; CFI $=.951 ; \mathrm{RMSEA}=.037[.030, .044] ; \mathrm{SRMR}=.040)$.

Considerando que los estudios anteriores no reportan las propiedades de la invarianza factorial del instrumento y que otras investigaciones sobre el perfeccionismo han reportado diferencias en la relación de la estructura del perfeccionismo en hombres y mujeres (Klibert et al., 2015; Stoeber, 2018), mientras que en otros estudios no presentaron variaciones según sexo en adolescentes (p.ej., Gavino et al., 2017; Vicent et al., 2019), se considera pertinente estimar la equivalencia de medicion del instrumento para precisar si la medida es invariante, y permita interpretar los hallazgos de manera general en ambos sexos.

En relación a las evidencias de validez con otras variables, en los estudios instrumentales previos de la EPI no evidenciaron asociación con variables afectivas, a pesar de que Bandura (1997) señala que cuando las personas enfrentan situaciones donde las exigencias para su cumplimiento son elevadas y no hay control sobre los pensamientos negativos, las personas pueden presentar sintomatología asociada a la depresión y ansiedad, por lo 
tanto, tales rasgos del perfeccionismo afectan los pensamientos y sentimientos. En consecuencia, las personas perfeccionistas pueden verse afectadas por los síntomas depresivos (Arana et al., 2017; Oros et al., 2017) cuya evidencia ha sido sistematizado por Limburg et al. (2017), sobre los vínculos del perfeccionismo con diversos trastornos emocionales y de ansiedad.

A su vez el estudio de Dunkley et al. (2012) demostró que los sujetos con rasgos de perfeccionismo se ven a sí mismo como defectuosos y deficientes, además presentan sentimientos de inferioridad, baja autoestima y afecto negativo vinculados a la autodesvalorización (Stoeber, 2018), debido a la incapacidad de satisfacción de sus propias demandas excesivamente altas.

Por lo tanto, en base a los planteamientos teóricos y empíricos, es menester hipotetizar asociaciones directas e inversas con dimensiones vinculadas a los síntomas depresivos a fin de proveer mayores evidencias de validez que las proporcionadas por los estudios de enfoque analítico-factorial.

Finalmente, considerando la importancia de una medida sobre el perfeccionismo en las diferentes áreas del ámbito académico y personal del estudiante y la necesidad de contar con instrumentos de medición que cumplan con los Standards for Educational and Psychological Testing (AERA, APA, y NCME, 2014), esta investigación tiene como objetivo evaluar las propiedades psicométricas de la adaptación de la EPI en adolescentes peruanos, tanto por su estructura interna como por su relación con variables de dominio afectivo.

\section{Método}

\section{Diseño}

Esta investigación es de tipo instrumental, debido a que analizaremos las propiedades psicométricas y la adaptación de un instrumento psicológico (Ato, López y Benavente, 2013) mediante el AFC, equivalencia métrica y validez de relación con los síntomas depresivos.

\section{Participantes}

La muestra estuvo compuesta por 917 estudiantes de dos instituciones educativas nacionales de la ciudad Lima Metropolitana, lo cuales fueron 464 varones y 453 mujeres de educación secundaria cuyas edades fueron entre 13 a 18 años $(M=15.241, D T=1.020)$. Se utilizó una calculadora de tamaño a priori para el modelo de ecuación estructural para calcular el tamaño de muestra necesario para el análisis factorial confirmatorio (AFC). Dado el número de variables observadas y latentes en el modelo, el tamaño mínimo del efecto previsto de 0.30 , un nivel de probabilidad de 0.05 y potencia estadística de 0.95 (Ramos-Vera, 2020; Soper, 2020).

\section{Instrumento}

Escala de Perfeccionismo Infantil (EPI; Oros, 2003). Se trata de una escala de autoinforme de 16 ítems que evalúan reactivos asociados al perfeccionismo en niños, compuesto por dos dimensiones: Autodemandas (ítems 1 al 8) y reacciones frente al fracaso (9 al 16). La escala se contesta según un rango de respuestas de tres puntos, que varía entre 1 («No») 2 («A veces») y 3 («Si»). Se ha proporcionado evidencia sobre sus excelentes propiedades psicométricas (Ventura-León et al., 2018). En el presente estudio se obtuvo una fiabilidad de alfa: $a=.87$.

Reynolds Adolescent Depression Scale (RADS; Reynolds, 1987). Se utilizó la versión peruana de Ugarriza y Escurra (2002). Es un instrumento compuesto por 30 ítems que evalúa los síntomas de depresión en adolescentes de 13 a 18 años, Su nivel de medición es de tipo Likert y cuenta con un rango de respuestas de cuatro puntos, que varían entre 1 («Casi nunca») y 4 («Casi Siempre»). Esta medida cuenta con seis dimensiones: Desmoralización y desesperanza; emociones negativas; desvalorización; síntomas somáticos y ansiedad, en donde a partir de mayor puntaje se estima un mayor nivel de depresión. Para la presente investigación se obtuvo una fiabilidad de alfa: $\alpha=.87$.

\section{Procedimiento}

Antes de realizar el proceso de adaptación se revisó las directrices para la adaptación de los test (Muñiz, Elosua y Hambleton, 2013) para su uso en una muestra adolescente peruana. Aunque se reportó una versión peruana previa de la EPI (Ventura-León et al., 2018) la redacción de los ítems es la misma a la versión original de Oros (2003), y considerando que se pretende adaptar el instrumento en una muestra de mayor edad a diferencia de las investigaciones previas de la EPI, se decidió evaluar este proceso. A través de un estudio piloto de 10 estudiantes, con la finalidad de evaluar la claridad y pertenencia de los ítems. Posteriormente se valoró la adaptación cultural a través de expertos definidos como profesionales con experiencia en el tema (Escobar y Cuervo, 2008), lo que permitió modificar 11 de los 16 contenidos de los reactivos con la finalidad de una mejor compresión de los ítems para la población adolescente considerando que los temas culturales pueden afectar las interpretaciones (Escobar y Cuervo, 2008; Van de Vijver y Hambleton, 1996). Se obtuvieron valores de V de Aiken 
aceptables, en donde se lograron valores mayores al mínimo establecido ([ $\geq .70]$; Aiken, 1985; Escurra, 1988). Por otro lado, en relación con la escala Likert, se optó por aumentar el número de opciones de respuesta de 3 a 5 debido a que mejoran la fiabilidad y calidad de respuestas de los estudios instrumentales ( $\mathrm{p}$ ej., Calleja et al., 2019; Xu y Leung, 2018). Después de evidenciar la validez de contenido, se aplicó la encuesta en una muestra única de 917 adolescentes.

Se aprovechó los talleres realizados en los centros educativos para explicarles el proyecto de investigación y los fines académicos a los responsables de las instituciones educativas, los cuales firmaron un consentimiento informado. Posteriormente, durante la ejecución, los estudiantes voluntariamente firmaron un asentimiento informado, el cual señala los objetivos del estudio y la confidencialidad de los participantes. Adicionalmente se requirió el permiso a los directores de ambas instituciones educativas mediante una solicitud formal dando a conocer el objetivo de la investigación. Estos se encargaron de informar los objetivos de la investigación tanto a los padres o tutores como a los estudiantes, con el fin de obtener el consentimiento y asentimiento de los mismos, así como dispone el artículo 24 del código de ética (Colegio de Psicólogos del Perú, 2017). El instrumento se respondió de manera voluntaria y anónima por 917 evaluados, se incluyeron las respuestas de todos los participantes.

\section{Análisis de datos}

Mediante el programa R (psych package, versión 3.4.2) se llevó a cabo los análisis de los resultados del presente estudio. El análisis estadístico tuvo en cuenta evaluar los items de forma preliminar teniendo en consideración los coeficientes de curtosis y asimetría (Hair et al., 1995) para comprobar el supuesto o no de normalidad multivariada, incluyendo el coeficiente de Mardia < 70 (Mardia, 1970). Se consideró el análisis de correlación ítem-test $(i-t)$ para el posible retiro de ítems en el caso de que sea $i-t<20$ y presente posible multicolinealidad (Kline, 2010).

Se procedió a utilizar el AFE y AFC considerando el estudio previo de Ventura-León et al. (2018). En primera instancia, se estimó el Análisis Factorial Exploratorio (AFE), técnica estadística que reduce datos por la correlación entre sus elementos. Se tuvo en cuenta los criterios de Hair, Black, Babin y Anderson (2014) con una muestra mínima de diez participantes por ítems del instrumento. Se examinaron las medidas de adecuación muestral: coeficiente de Káiser-Meyer (Káiser, 1970) y la prueba de esfericidad de Bartlett (Bartlett, 1950). La estimación del número de factores fue mediante el análisis paralelo (Horn, 1965) y el método de extracción fue mínimos cuadrados no ponderados; debido a que la distribución de los datos, en especial la multivariada, es no normal (Costello y Osborne, 2005).

Después de haber examinado las características descriptivas de los ítems y el AFE, se procedió a evaluar la estructura interna mediante al AFC. En dicho procedimiento se utilizaron diferentes índices de ajuste para evaluar los modelos: RMSEA ( $\leq .06)$, CFI ( $\geq .95)$, SRMR ( $\leq$ $.06)$ y la prueba general $x^{2}$ corregida por la falta de normalidad de las variables ( $\mathrm{SB} \chi^{2}$; Satorra y Bentler, 2001).

La consistencia interna de la escala, se estimó mediante el coeficiente alfa y omega ( $a$ y $\omega \geq .70$ ) (Hair et al., 2014). Y se evaluó la invarianza factorial del instrumento en función al sexo (Byrne, 2008).

La evidencia de validez concurrente se obtuvo a partir de las correlaciones entre los factores de la medida del perfeccionismo y los síntomas depresivos estimados mediante el coeficiente de correlación de Pearson ( $r$ ), estas medidas pueden ser utilizadas considerando la naturaleza multidimensional de la EPI y la DARS evaluadas mediante el análisis factorial (McNeish y Wolf, 2020).

Finalmente se realizó el análisis de red a través del programa Jasp versión 0.11 (Jasp Team, 2019) para evaluar las características de las relaciones sistémicas entre las variables de estudio a través del algoritmo de Fruchterman-Reingold (Fruchterman y Reingold, 1991). Los parámetros que se consideraron son el estimador Ebiclasso (Chen y Chen, 2008; Friedman et al., 2008) que permite generar una red estable basado en las correlaciones parciales regularizadas y de fácil interpretación con un índice de estimación de 0.5 (Foygel y Drton, 2010) recomendable para crear una red parsimoniosa con mayor estabilidad (Epskamp y Fried, 2018).

En la red se presentan nodos, estos representan las variables de estudio que se conectan a través de los bordes (líneas), que presentan un peso que determina la intensidad de la relación, esto se evidencia gráficamente por el grosor de la línea que relacionan a los nodos, de mayor interés en la investigacion psicológica y psiquiatrica (Ramos-Vera, 2021a; Ramos-Vera \& Serpa, 2021). Este método multivariante incluye índices de centralidad para estimar la interconexión en la estructura de la red (Opsahl et al., 2010), en donde el indicador de mayor centralidad presentaría una influencia más fuerte con los demás componentes de la red (Epskamp y Fried, 2018). Estos índices son la magnitud de conectividad con los otros nodos (centralidad de fuerza), la proximidad entre todas las variables de la red (centralidad de proximidad), esta variable presenta un mayor poder predictivo en los demás, y la frecuencia de las conexiones que tiene cada nodo entre la ruta más corta entre los demás pares de conexiones posibles (centralidad de intermediación) (Costantini y Perugini, 2017). 


\section{Resultados}

Como resultado de la revisión de los ítems descrita en la sección de «procedimientos» se ha decidido modi- ficar la redacción de 11 ítems de la versión de la EPI para su uso en población adolescente. En la Tabla 1 se puede observar la versión original y la versión adaptada actual.

Tabla 1. Ítems originales y adaptados

\begin{tabular}{cll}
\hline ítem & \multicolumn{1}{c}{ Versión original (Oros, 2003) } & \multicolumn{1}{c}{ Versión Adaptada para adolescentes } \\
\hline 1 & Necesito ser el mejor & *Necesito ser el/la mejor \\
2 & Tengo que ser el mejor alumno & *Tengo que ser la/el mejor alumno(a) \\
3 & Debo ganar siempre & *Debo ganar siempre en las actividades que realizo \\
4 & No puedo cometer errores & No puedo cometer errores \\
5 & Mis trabajos deben ser mejores que los demás & Mis trabajos deben ser mejores que los demás \\
6 & No debo perder cuando juego con mis amigos & $*$ No debo perder cuando compito con mis amigos \\
7 & Debo ser el mejor de la clase & $*$ Debo ser el/la mejor de la clase \\
8 & Debo ser el primero en terminar las tareas escolares & $*$ Debo ser el/la primero(a) en terminar las tareas escolares \\
9 & Cuando pierdo me siento mal & $*$ Me siento mal cuando pierdo en una competencia académica \\
10 & Pienso mucho en las equivocaciones que tuve & $*$ Me preocupo mucho por mis equivocaciones y fracasos \\
11 & Me siento muy mal cada vez que pierdo en algún juego o deporte & $*$ Me siento muy mal cuando pierdo al jugar o practicar deporte \\
12 & Me cuesta perdonarme cuando me equivoco & Me cuesta perdonarme cuando me equivoco \\
13 & Me enojo cuando no logro lo que quiero & Me enojo cuando no logro lo que quiero \\
14 & Me critico mucho a mí mismo & $*$ Me critico mucho a mí mismo(a) cuando cometo equivocaciones \\
15 & Me siento culpable cuando cometo algún error & $*$ Tengo sentimientos de culpa por mis errores y fracasos \\
16 & Me insulto cada vez que cometo un error & Me insulto cada vez que cometo un error \\
\hline
\end{tabular}

Nota. * Ítems adaptados.

\section{Análisis preliminar de los items}

Se obtuvieron valores dentro del rango estimado \pm 1.5 (Muthén y Kaplan, 1985, 1992) en asimetría y curtosis; aunque, la curtosis multivariada de Mardia (1970) obtuvo puntuaciones $>70\left(G^{2}=332.92, p<.001\right)$. En base a esto, se atenuaron los datos mediante el estimador corregido al Chi cuadrado por Satorra-Bentler (Satorra y Bentler, 2001) (para mayor detalle ver Tabla 2). La discriminación de los ítems arrojó valores aceptables entre los reactivos con valores de .37 a .60 respectivamente.

\section{Análisis factorial exploratorio (AFE)}

Para realizar el análisis factorial exploratorio se consideró las medidas de adecuación muestral, a través del $\mathrm{KMO}=.902 \mathrm{y}$ el test de esfericidad de Bartlett, que resultaron ser estadísticamente significativos $(p<.001)$. El Análisis Paralelo (AP) realizado sugirió la extracción de tres factores que subyacen a los dieciséis ítems. Los tres factores explican el $57.91 \%$ de la varianza del constructo del perfeccionismo (la denominación de los factores es como sigue: autodemandas [AD], malestar-preocupación [MP] y autocastigo [AU]). La organización de los ítems se muestra en la Tabla 3, donde se observa cargas factoriales superiores a .40 (Kline, 2010) (ver Tabla 3 y 4). En cuanto a los índices de consistencia interna alfa y omega para los tres factores de la escala (autodemandas, malestar-preocupación y autocastigo) fluctuaron entre .68 y .87 .

\section{Análisis factorial confirmatorio (AFC)}

Los resultados explorados de una estructura de tres dimensiones fueron posteriormente evaluados mediante AFC que reportó diferentes índices de bondad de ajuste óptimos $\left(\mathrm{SB} \chi^{2} / g l=0.508, \mathrm{CFI}=.979\right.$, RMSEA [IC 90 $\%]=.055[.049-.061] ; \mathrm{SRMR}=.056)$

\section{Invarianza factorial}

Para la determinación de la invarianza factorial (ver Tabla 5), se evaluó comenzando a través de la invarianza 
Tabla 2. Estadísticos descriptivos de la adaptación de la EPI en adolescentes peruanos

\begin{tabular}{|c|c|c|c|c|c|}
\hline Ítems & $M$ & $D T$ & $g 1$ & $g^{2}$ & $i-t$ \\
\hline $\mathrm{P} 1$ & 3.358 & 1.079 & -0.162 & -0.452 & .480 \\
\hline $\mathrm{P} 2$ & 3.298 & 1.029 & -0.102 & -0.401 & .464 \\
\hline P3 & 3.289 & 1.071 & -0.140 & -0.629 & .527 \\
\hline $\mathrm{P} 4$ & 2.990 & 1.161 & 0.036 & -0.741 & .498 \\
\hline P5 & 3.049 & 1.110 & -0.044 & -0.627 & .561 \\
\hline P6 & 2.733 & 1.147 & 0.231 & -0.646 & .526 \\
\hline P7 & 2.909 & 1.055 & 0.041 & -0.419 & .566 \\
\hline P8 & 2.731 & 1.043 & 0.197 & -0.425 & .548 \\
\hline P9 & 2.671 & 1.019 & 0.103 & -0.162 & .517 \\
\hline $\mathrm{P} 10$ & 3.354 & 1.032 & -0.218 & -0.488 & .501 \\
\hline P11 & 2.754 & 1.154 & 0.243 & -0.627 & .499 \\
\hline $\mathrm{P} 12$ & 2.671 & 1.175 & 0.333 & -0.664 & .451 \\
\hline P13 & 3.087 & 1.146 & 0.042 & -0.646 & .551 \\
\hline P14 & 2.859 & 1.207 & 0.174 & -0.822 & .535 \\
\hline $\mathrm{P} 15$ & 2.888 & 1.185 & 0.139 & -0.763 & .496 \\
\hline $\mathrm{P} 16$ & 2.138 & 1.241 & 0.901 & -0.179 & .365 \\
\hline \multicolumn{2}{|c|}{ Kurtosis de Mardia } & 332.925 & & & \\
\hline
\end{tabular}

Nota. M: Media, DT: desviación típica, $g 1$ : Asimetría, $g^{2}$ : Curtosis, $i-t$ : correlación ítem-test.

Tabla 3. Matriz del factor de la adaptación de la EPI en adolescentes peruanos rotado con el método Promin

\begin{tabular}{|c|c|c|c|c|}
\hline Ítem & $\mathrm{F} 1$ & $\mathrm{~F} 2$ & F3 & $h^{2}$ \\
\hline $\mathrm{P} 1$ & .680 & .110 & .315 & .464 \\
\hline P2 & .716 & .094 & .246 & .532 \\
\hline P3 & .695 & .137 & .407 & .491 \\
\hline P4 & .493 & .285 & .424 & .290 \\
\hline P5 & .702 & .191 & .444 & .503 \\
\hline P6 & .560 & .227 & .490 & .371 \\
\hline P7 & .830 & .150 & .332 & .702 \\
\hline P8 & .711 & .167 & .415 & .511 \\
\hline P9 & .403 & .347 & .620 & .396 \\
\hline $\mathrm{P} 10$ & .373 & .378 & .561 & .330 \\
\hline P11 & .273 & .457 & .699 & .499 \\
\hline $\mathrm{P} 12$ & .161 & .631 & .494 & .422 \\
\hline P13 & .279 & .625 & .609 & .476 \\
\hline P14 & .212 & .814 & .507 & .665 \\
\hline P15 & .165 & .814 & .465 & .663 \\
\hline P16 & .074 & .687 & .333 & .483 \\
\hline
\end{tabular}

Varianza total explicada: $57.915 \%$

$\mathrm{KMO}=.902$, Bartlett: $x^{2}(120)=5856.3, p<.001$

Nota. Método de extracción: mínimos cuadrados no ponderados, $h^{2}$ : comunalidades. 
Tabla 4. Cargas factoriales y correlación de factores de la adaptación de la EPI en adolescentes peruanos

\begin{tabular}{|c|c|c|c|}
\hline Ítem & $\mathrm{AD}$ & MP & $\mathrm{AU}$ \\
\hline $\mathrm{P} 1$ & .709 & & \\
\hline P2 & .748 & & \\
\hline P3 & .723 & & \\
\hline $\mathrm{P} 4$ & .579 & & \\
\hline P5 & .747 & & \\
\hline P6 & .645 & & \\
\hline P7 & .844 & & \\
\hline P8 & .754 & & \\
\hline P9 & & .641 & \\
\hline $\mathrm{P} 10$ & & .623 & \\
\hline P11 & & .628 & \\
\hline $\mathrm{P} 12$ & & & .728 \\
\hline P13 & & & .784 \\
\hline P14 & & & .871 \\
\hline P15 & & & .841 \\
\hline $\mathrm{P} 16$ & & & .725 \\
\hline $\mathrm{AD}$ & - & .585 & .299 \\
\hline MP & - & - & .691 \\
\hline
\end{tabular}

Nota. AD: Autodemandas, MP: malestar-preocupación, AU: autocastigo

Tabla 5. Invarianza factorial de la adaptación de la EPI en adolescentes peruanos

\begin{tabular}{|c|c|c|c|c|c|c|}
\hline Invarianza & $x^{2} / g l$ & $p$ & CFI & RMSEA & $\triangle \mathrm{CFI}$ & $\triangle \mathrm{RMSEA}$ \\
\hline Configural & $805.082 / 202$ & $<.001$ & .979 & .080 & - & - \\
\hline Métrica & $865.492 / 215$ & $<.001$ & .978 & .081 & .001 & .001 \\
\hline Interceptos & $956.305 / 260$ & $<.001$ & .976 & .075 & .002 & .006 \\
\hline Residual & $965.175 / 263$ & $<.001$ & .976 & .076 & .000 & .001 \\
\hline
\end{tabular}

Nota $x^{2}=$ Chi Cuadrado, gl: grados de libertad, CFI: Índice de ajuste Comparativo, RMSEA: Error Cuadrático Medio de Aproximación. $\triangle \mathrm{CFI}$ : Índice de ajuste Comparativo delta, $\triangle$ RMSEA: Error Cuadrático Medio de Aproximación delta

configuracional a partir del cual se genera los demás modelos con restricciones, este modelo presentó índices de ajuste de $(\mathrm{CFI}=.979$ y RMSEA $=.080)$ que permiten aceptar el modelo, se realizó posteriormente el modelo de invarianza métrica (M2) con adecuado ajuste de invarianza $(\Delta \mathrm{CFI}=.001$ y $\triangle \mathrm{RMSEA}=.001)$, estos resultados cumplen los parámetros requeridos de $\Delta \mathrm{CFI} \leq .01$ y $\Delta \mathrm{R}$ MSEA $\leq .015$ (Chen, 2007; Cheung \& Rensvold, 2002) que permiten continuar con este análisis. Posteriormente, se evaluó la invarianza de interceptos cumpliendo con los parámetros respectivos $(\Delta \mathrm{CFI}=.002$ y $\triangle \mathrm{RMSEA}=.006)$. Y finalmente se estimó la invarianza residual, que reportó una diferencia mínima con el modelo anterior con valores de $\Delta$ CFI $=.000$ y $\triangle$ RMSEA $=.001$ (Byrne, 2008).

\section{Análisis de evidencia de correlación entre perfeccionismo y sintomas depresivos}

La evidencia de validez convergente se evaluó en función de las relaciones con las variables externas. Los resultados se presentan en la Tabla 6, donde se verifican los índices de correlación de Pearson entre las subescalas de la EPI, y a su vez entre estas y las variables externas: Desmoralización y desesperanza, emociones negativas, anhedonia, desvalorización, síntomas somáticos y ansiedad.

Los resultados (ver tabla 6) indican una asociación moderada, positiva y significativa entre los factores de la escala de perfeccionismo. Con respecto a las variables externas, se observaron niveles de correlación, que va- 
Tabla 6. Correlación de Pearson entre escalas que componen la EPI y síntomas depresivos $(n=917)$

\begin{tabular}{lcccccccc}
\hline \multicolumn{1}{c}{ Invarianza } & 1 & 2 & 3 & 4 & 5 & 6 & 7 & 8 \\
\hline 1. Desmoralización y desesperanza & - & & & & & & & \\
2. Emociones negativas & $.641^{* * *}$ & - & & & & & & \\
3. Anhedonia & -.036 & -.013 & - & & & & & \\
4.Desvalorizacion & $.692^{* * *}$ & $.687^{* * *}$ & $-.137^{* * *}$ & - & & & & \\
5.Síntomas Somáticos & $.472^{* * *}$ & $.538^{* * *}$ & .030 & $.440^{* * *}$ & - & & & \\
6.Ansiedad & $.194^{* * *}$ & $.227^{* * *}$ & .031 & $.176^{* * *}$ & $.194^{* * *}$ & - & & \\
7.Autocastigo & $.455^{* * *}$ & $.471^{* * *}$ & -.053 & $.462^{* * *}$ & $.320^{* * *}$ & $.178^{* * *}$ & - & \\
8.Malestar-Preocupacion & $.263^{* * *}$ & $.240^{* * *}$ & .028 & $.196^{* * *}$ & $.167^{* * *}$ & $.258^{* * *}$ & $.514^{* * *}$ & - \\
9.Autodemandas & .064 & -.040 & .029 & -.030 & .044 & $.077^{*}$ & $.255^{* * *}$ & $.453^{* * *}$ \\
\hline
\end{tabular}

Nota. $* p<.05 ; * * p<.01 ; * * * p<.001$

rían de magnitud positiva moderada a fuerte entre los dominios de malestar-preocupación y autocastigo, con los componentes de desmoralización y desesperanza; emociones negativas; desvalorización; síntomas somáti$\cos$ y ansiedad. Estos resultados apoyan la expectativa teórica de evidencia de validez convergente. Finalmente, hay una asociación mínima entre la dimensión de autodemandas y el rasgo de ansiedad.

Aunque los indicadores de correlación de Pearson permiten estimar la evidencia de validez en función de la relación con variables externas, estas asociaciones son de orden bivariado y no permiten una mejor comprensión de la naturaleza compleja del perfeccionismo en la asociación con otros síntomas psicopatológicos. En vista de esta brecha, se realizó un análisis de red para investigar la estructura de asociación dinámica entre las variables empleadas en el presente estudio.

Los resultados se muestran en la Figura 1, donde se verifica la representación gráfica de la red, además de diferentes medidas de centralidad (ver Figura 2).

Las variables se organizaron de acuerdo con las magnitudes de las asociaciones entre sí, como se esperaba de
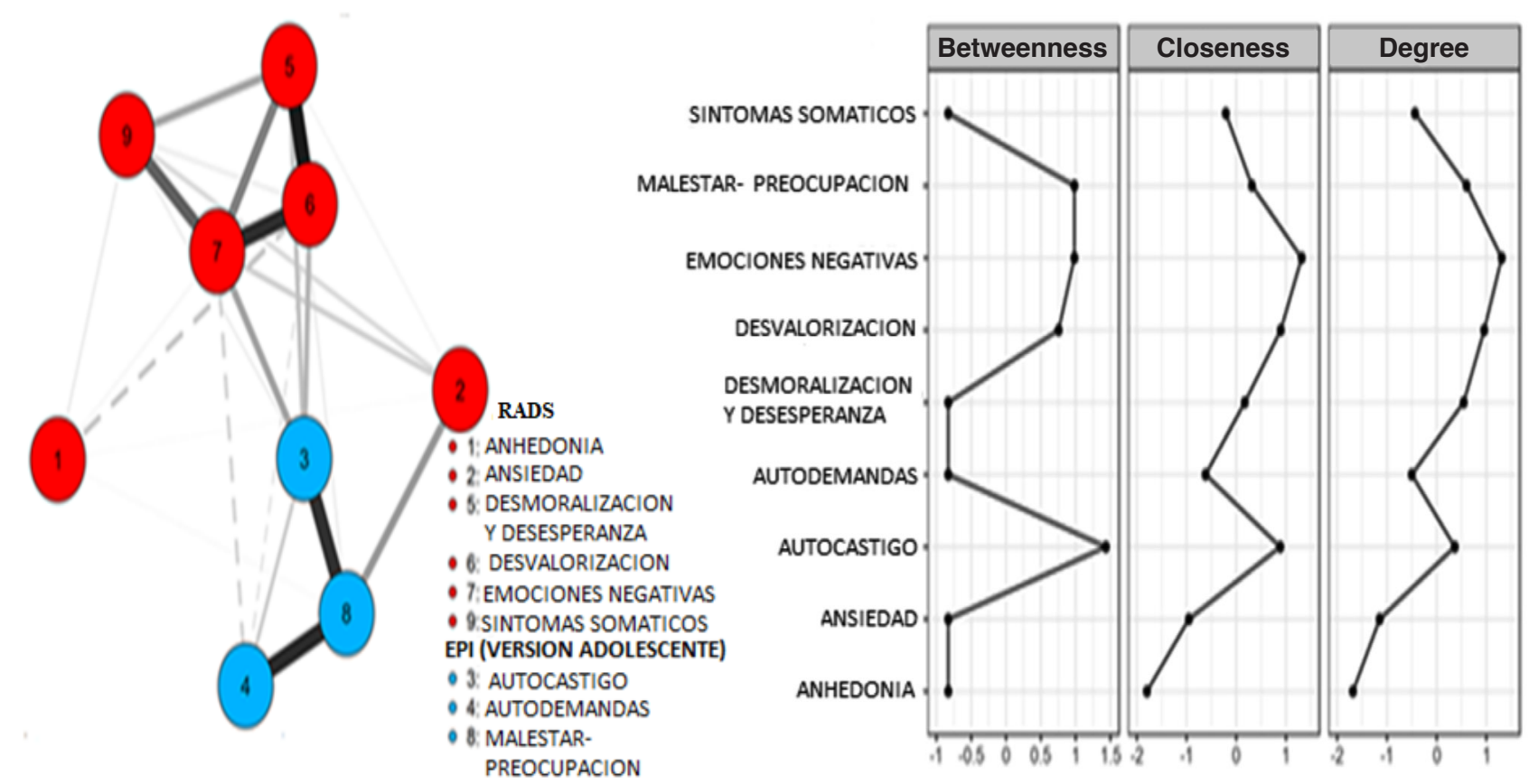

Nota: Cada uno representa una variable de estudio, las líneas continuas indican asociaciones positivas y las líneas puntiagudas representan relaciones negativas.

Figura 1. Representación gráfica de la red Figura 2. Índices de centralidad 
manera positiva, entre sus respectivas variables, solo la dimensión de anhedonia presenta correlación negativa con la desvalorización de la variable general de depresión (Tabla 6). En cuanto al factor de autodemandas, reporta vínculos negativos con los factores de desvalorización y emociones negativas. Sin embargo, se observa que las variables de perfeccionismo que ocupan más posiciones centrales en la red son el autocastigo, y malestar-preocupación, mientras que los dominios del RADS más destacables son el factor de emociones negativas. Estos componentes de la red presentan una mayor medida de intermediación, lo que sugiere que estas variables tienden a ser elementos nexo para conectar los demás pares de variables en la red fijando rutas más cortas. Tales componentes de la red y la desvalorización reportan mayores medidas de centralidad de cercanía, pues presentan distancias más cortas en la red, es decir, que su ubicación es más central en la red y permite estar más conectadas con todas las otras variables del sistema interactivo, por lo tanto, refieren un mayor potencial en la predicción de las demás variables; a su vez también reportan mayor índice de fuerza, debido a las altas medidas de asociación y al mayor número de interconexiones de red. El síntoma de anhedonia o pérdida de interés mostró los indicadores más bajos de centralidad en la red, lo que sugiere una menor relación con las demás medidas.

\section{Discusión}

Los niveles de perfeccionismo está aumentando en los últimos treinta años (Curran y Hill, 2019), y en especial los adolescentes que se encuentran en una etapa de desarrollo importante en la construcción de la personalidad, que pueden verse envueltos en la «paradoja del perfeccionismo», impulsados por el entorno a perseguir objetivos elevados en su vida personal proponiéndose exigencias elevadas por lograr un rendimiento perfecto que pueden perjudicar su salud mental (Bieling et al., 2004). Por lo tanto, el objetivo consistió en adaptar la escala de perfeccionismo infantil de Oros (2003) en una muestra de adolescentes peruanos, así como determinar sus propiedades psicométricas. En ese sentido, la adaptación de esta medida en adolescentes peruanos indica que debe ser interpretada como una escala trifactorial y que la fiabilidad de las puntuaciones resulta buenas medidas del constructo examinado.

La validación basada en la estructura interna se realizó mediante el modelo factorial. Se realizó un procedimiento similar al estudio previo de la EPI de Ventura-León et al. (2018), en donde se exploró la estructura del instrumento en primera instancia mediante el AFE que estimó tres factores (autodemandas [AD], malestar-preocupación [MP] y autocastigo [AU]). Y, en segunda instancia se procedió a evaluar a través del AFC, obteniendo índices de ajuste óptimo para la adaptación en adolescentes de la EPI. Cabe señalar que estos hallazgos no fueron esperados ya que no concuerdan con la versión original (Oros, 2003) y peruana (Ventura et al., 2018) de dos dimensiones y difieren de la adaptación venezolana (Aguilar y Castellanos, 2017). Los hallazgos refieren que la primera dimensión mantiene su estructura, mientras el otro factor denominado reacciones ante el fracaso reportó la división en dos dominios.

Los hallazgos para la determinación de 3 dimensiones fueron obtenidos a partir del análisis paralelo que es el método más preciso considerando que los datos no se distribuyeron con normalidad (Li et al., 2020) como parte del AFE que estimaron un 58\% de la varianza del contructo del perfeccionismo, siendo de mayor medida en comparación de las investigaciones instrumentales previas de la EPI como el estudio original de Oros (2003), el cual solo explicaba el $41 \%$ de la varianza total, de igual manera en la versión peruana previa $(44 \%$ de la varianza).

Los dos dominios del factor reacciones ante el fracaso no es algo nuevo pues en el estudio de Smith et al. (2016) se señala que tales reacciones se conceptualizan en un factor de orden superior denominado perfeccionismo autocrítico, compuesto por cinco facetas, dos de los cuales están vinculados a la preocupación-malestar y al autocastigo (Casale et al., 2020; Sherry et al., 2018; Smith et al., 2016). Siendo probable que los adolescentes tengan la capacidad de distinguir estos dos dominios a diferencia de los niños considerando los estudios previos de la EPI.

Por otro lado, el análisis de la invariabilidad según sexo, permite fortalecer las propiedades psicométricas del EPI no encontrándose diferencias en la conceptualización del constructo entre varones y mujeres de acorde con los estándares propuestos por The Standards for Educational and Psychological Testing (AERA, APA, y NCME, 2014). Hubo dos investigaciones instrumentales que evaluaron el perfeccionismo en adolescentes de España (Gavino et al., 2017) y Ecuador (Vicent et al., 2020) que determinaron la invarianza factorial según sexo por lo que la presente investigación también demuestra que tal equivalencia del constructo también ocurre en adolescentes peruanos.

Adicionalmente, se reportó evidencia de validez basada en la relación con variables externas, de orden convergente, vinculado a los síntomas depresivos. Los resultados obtenidos corroboran las hipótesis teóricas y son consistentes con otros estudios que utilizaron estas 
variables (Arana et al., 2017; Limburg et al., 2017; Oros et al., 2017).

Los hallazgos respaldan la expectativa de que los síntomas depresivos son de mayor riesgo en adolescentes que presentan rasgos perfeccionistas de preocupación-malestar, y conductas de autocastigo por los errores y fallos (Gavino et al., 2017). Cuyo aporte aún no se ha evidenciado en otros estudios instrumentales o correlacionales ya que hasta la fecha solo se han reportado relaciones estadísticas entre medidas generales de perfeccionismo y depresión, a diferencia de nuestros hallazgos que precisan relaciones significativas entre los factores de estos conceptos psicológicos. Específicamente, las correlaciones de Pearson fueron significativas entre la dimensión autocastigo y los síntomas depresivos siendo de mayor magnitud en comparación con la dimensión malestar-preocupación $\mathrm{y}$, con respecto a la dimensión autodemandas que sólo reporto relación significativa con la dimensión ansiedad. El síntoma depresivo de anhedonia fue el único que no registró una asociación significativa con las dimensiones de la medida del perfeccionismo.

Es el primer estudio instrumental del perfeccionismo en adolescentes (García-Fernández et al., 2016) que realiza un análisis de red hasta donde sabemos, el cual permite una comprensión dinámica de las asociaciones entre varias variables (p.ej., Ramos-Vera, 2021b). Desde el modelo de red se reportó asociaciones negativas significativas de los síntomas depresivos de emociones negativas y la desvalorización con la faceta perfeccionista de autodemandas asociado al perfeccionismo orientado hacia el esfuerzo (Stoeber, 2018). Esta dimensión del perfeccionismo presenta un mecanismo adaptativo caracterizado por un mejor ajuste psicológico, contrario a los otros dominios del perfeccionismo (Stoeber y Otto, 2006) que ha sido corroborado previamente (Dunkley et al., 2012; Stoeber y Gaudreau, 2017).

El modelo sistémico de red estimó como elementos centrales a los dominios: emociones negativas y autocastigo, que representan los síntomas «puente», es decir, que refieren la característica comórbida de conectar los síntomas depresivos con los síntomas del perfeccionismo (Hofmann et al., 2016). Siendo de gran importancia para futuras intervenciones clínicas desde el enfoque de red (p.ej., Blanco et al., 2020), donde a partir de la reducción de ambos elementos centrales se logre disminuir la activación dinámica de los demás dominios de la red.

Las conexiones de mayor medida en la red: son entre los síntomas depresivos de desvalorización y emociones negativas con el componente de autocastigo, pues según Stoeber (2018) refiere que las personas perfeccionistas pueden ser más vulnerables a estos síntomas depresivos.
Tales evidencias del enfoque de red permiten precisar que esta faceta perfeccionista presenta una asociación causal con los correlatos depresivos, lo cual ante un abordaje conjunto a estos síntomas con mayor covarianza en la red permitiría aumentar la eficacia clínica. Debido a que la reducción de estos correlatos psicopatológicos puede disminuir la resistencia al cambio en las intervenciones de salud mental (Lloyd et al., 2015). En especial las emociones negativas de ánimo deprimido, que ya ha sido reconocido como uno de los componentes de mayor activación en las redes de síntomas depresivos (Ramos-Vera et al., 2021).

El dominio de autodemandas presentó conexiones negativas con los síntomas depresivos mencionados. Las asociaciones ambivalentes significativas refuerzan la complejidad adaptativa e inadaptativa del perfeccionismo (Bieling et al., 2004; Flett y Hewitt, 2019). Por lo cual, las características del perfeccionismo además de presentar riesgos en la salud mental también pueden ser beneficiosas, ya que la faceta de las autodemandas está asociado a resultados positivos en base a la motivación por el logro personal y un rendimiento autoeficaz (Dunkley et al., 2012; Slade y Owens, 1998).

Actualmente existen propuestas psicológicas que evidencian la supresión de los riesgos asociados a la personalidad perfeccionista (Starley, 2018; Stoeber y Gaudreau, 2017), que destacan su vínculo con la ansiedad. De manera similar este estudio reportó una relación positiva entre la ansiedad y el dominio de malestar-preocupación. Ambos dominios pueden ser intervenidos y direccionados desde un enfoque positivo, donde tal carácter perfeccionista es una forma de hacer frente a la ansiedad que surge de una necesidad insatisfecha. A partir de esta perspectiva se puede plantear una intervención centrada en soluciones y orientada en el pensamiento sistémico de los rasgos perfeccionistas (Starley, 2018).

Cuyo abordaje favorecería al entorno y las personas cercanas de los adolescentes perfeccionistas a adaptarse a las dificultades vinculadas al perfeccionismo (p.ej., reacciones negativas), enfocándose como una habilidad de afrontamiento que necesita direccionarse de manera positiva considerando sus características adaptativas asociadas a un buen ajuste (Starley, 2018), evidenciado en las asociaciones negativas entre los dominios de red: autodemandas, emociones negativas y la desvalorización.

En cuanto a las limitaciones del estudio, en primer lugar, debido a razones de accesibilidad, solo se incluyeron los escolares de instituciones educativas nacionales, mientras que aquellos de las privadas no fueron considerados. Debido a ello existe la probabilidad de que los participantes en esta investigación sean los que obtuvie- 
ron mayores niveles de perfeccionismo, sesgando así los resultados, en consecuencia, se recomienda ampliar el tamaño muestral y realizar investigaciones con muestras probabilísticas. De esta manera, la generalización de los resultados queda limitada a las características de los participantes. En segundo lugar, la medición de la EPI se realizó transversalmente, por lo que estudios longitudinales brindarían mayor evidencia de validez predictiva. Por último, realizar investigaciones en las otras regiones del Perú, dado que los participantes en esta investigación corresponden solamente a la capital, Lima.

La principal fortaleza fue la inclusión del modelo de red, debido a que muchos estudios psicométricos todavía no utilizan este procedimiento estadístico que garantiza una mejor información técnica porque estima las asociaciones después del control multivariado de todas las variables (Epskamp y Fried, 2018), desde este modelo dinámico permite reforzar dinámicamente la complejidad de los correlatos del perfeccionismo y los síntomas depresivos en adolescentes desde la complejidad de sistemas de red de comorbilidad (Ramos-Vera, 2021c). Otra fortaleza a tomar en cuenta es la equivalencia de medición según sexo que fortalece las propiedades psicométricas de la adaptación para adolescentes de la EPI.

Se recomienda, de todos modos, ampliar los estudios psicométricos en diversas poblaciones estudiantiles. En este sentido, investigaciones futuras deberían avanzar en el estudio de la invarianza factorial del instrumento según grupos de edad, nivel socioeconómico, grupo étnico, etc., con el fin de garantizar la comparabilidad y la equivalencia del constructo del perfeccionismo en adolescentes. También es importante el uso de otros enfoques como el factor Bayes (Ramos-Vera, 2020; Ramos-Vera y Motta, 2021). Para evaluar las relaciones bivariadas que refuercen las evidencias de validez convergente y en la estimación de la fuerza probatoria en la comparacion de puntuaciones de test segun grupos categoricos (p.ej., Nuñez et al., 2021).

En conclusión, los resultados en cuanto a los aspectos evaluados de la confiabilidad y las evidencias de validez consideradas en este estudio, indican que la adaptación para adolescentes de la EPI muestra excelentes propiedades psicométricas para su evaluación en el contexto peruano. El empleo de medidas de autoinformes como la presente medida en estudios a escala nacional, dada su fácil y rápida administración, representa una línea de investigación altamente prometedora.

\section{Conflictos de intereses}

Los autores declaran no tener conflictos de intereses.

\section{Referencias}

AERA, APA, NCME. (2014). Standards for Educational and Psychological Testing. American Educational Research Association, American Psychological Association, National Council on Measurement in Education. Recuperado de https:// www.apa.org/science/programs/testing/standards.

Aguilar, L. A. y Castellanos, M. Y. (2017). Validación psicométrica de una escala de perfeccionismo infantil en niños venezolanos. Psicoespacios, 11(18), 9-42.

Aiken, L. R. (1985). Three coefficients for analyzing the reliability and validity of ratings. Educational and psychological measurement, 45(1), 131-142. https://doi.org/10.1177/0013164485451012

Arana, F. G., Rice, K. G. \& Ashby, J. S. (2017). Perfectionism in Argentina and the United States: Measurement Structure, Invariance, and Implications for Depression. Journal of Personality Assessment, 100(2), 219-230. https://doi.org/10.1 080/00223891.2017.1296845

Ato, M., López, J. J. y Benavente, A. (2013). Un sistema de clasificación de los diseños de investigación en Psicología. Análisis de Psicología, 29(3), 1038-1059.

Bandura, A. (1977). Self-efficacy: toward a unifying theory of behavioral change. Psychological Review, 84(2), 191-215. https://doi.org/10.1037/0033-295X.84.2.191

Bartlett, M. S. (1950). Tests of significance in factor analysis. British Journal of Mathematical and Statistical Psychology, 3(2), 7785. https://doi.org/10.1111/j.2044- 8317.1950.tb00285.x

Bieling, P. J., Israeli, A.L. \& Antony, M. M. (2004). Is perfectionism good, bad, or both? Examining models of the perfectionism construct. Personality and Individual Differences, 36(6), 1373-1385. https://doi.org/10.1016/S0191-8869(03)00235-6

Blanco, I., Contreras, A., Chaves, C., López-Gómez, I., Hervás, G. \& Vázquez, C. (2020). Positive interventions in depression change the structure of well-being and psychological symptoms: A network analysis. The Journal of Positive Psychology, 1-6. https://doi.org/10.1080/17439760.2020.1789696

Byrne, B. M. (2008). Testing for multigroup equivalence of a measuring instrument: A walk through the process. Psicothema, 20(4), 872-882.

Calleja, N., Reskala, F. J., Rivera-Fong, L. y Buenrostro, D. (2019). Efecto del número de opciones de respuesta en las propiedades psicométricas de cuatro escalas psicosociales. Revista de Psicología y Ciencias del Comportamiento de la UACJS, 10(2), 100-113. https://doi.org/10.29059/rpcc.20191126-94

Casale, S., Fioravanti, G., Rugai, L., Flett, G. L. \& Hewitt, P. L. (2019). What lies beyond the superordinate trait perfectionism factors? The perfectionistic self-presentation and perfectionism cognitions inventory versus the big three perfectionism scale in predicting depression and social anxiety. Journal of personality assessment, 102(3), 370-379. https://doi.org/10.1080/0022389 1.2019 .1573429

Chen, F. (2007). Sensitivity of Goodness of Fit Indexes to Lack of Measurement Invariance, Structural Equation Modeling. A Multidisciplinary Journal, 14(3), 464-504. https://doi. org/10.1080/10705510701301834

Chen, J. \& Chen, Z. (2008). Extended Bayesian information criteria for model selection with large model spaces. Biometrika, 95(3), 759-771. https://doi.org/10.1093/biomet/asn034 
Cheung, G. \& Rensvold, R. (2002). Evaluating goodness-of-fit indexes for testing measurement invariance. Structural Equation Modeling, 9(2), 233-255. https://doi.org/10.1207/ S15328007SEM0902_5

Colegio de psicólogos del Perú (2017). Código de ética y deontología. Recuperado de https://www.cpsp.pe/documentos/ marco_legal/codigo_de_etica_y_deontologia. pdf?fbclid=IwAR20yCb5yoFa0d5-OGKXutIfnbVd7a_UHwrE4b50--B3xooGQ39-FFYt3Y

Costantini, G. \& Perugini, M. (2017). Network analysis for psychological situations. In D. C. Funder, J. F. Rauthmann, \& R. A. Sherman (Eds.), The Oxford Handbook of psychological situations. Oxford University Press.

Costello, A. B. \& Osborne, J. W. (2009). Best practices in exploratory factor analysis: Four recommendations for getting the most from your analysis. Pan-Pacific Management Review, 12(2), 131-146. https://doi.org/10.7275/jyj1-4868

Curran, T. \& Hill, A. P. (2019). Perfectionism is increasing over time: A meta-analysis of birth cohort differences from 1989 to 2016. Psychological Bulletin, 145, 410-429. https://doi. org/10.1037/bul0000138

Davis, M. C. \& Wosinski, N. L. (2011). Cognitive Errors as Predictors of Adaptive and Maladaptive Perfectionism in Children. Journal of Rational-Emotive \& Cognitive-Behavior Therapy, 30(2), 105117. https://doi.org/10.1007/s10942-011-0129-1

Dunkley, D. M., Berg, J.-L. \& Zuroff, D. C. (2012). The role of perfectionism in daily self-esteem, attachment, and negative affect. Journal of Personality, 80(3), 633-663. https://doi. org/10.1111/j.1467-6494.2011.00741.x

Epskamp, S. \& Fried, E. (2018). A tutorial on regularized partial correlation networks. Psychological Methods, 23(4), 617-634. https://doi.org/10.1037/met0000167

Escobar, P. J. y Cuervo, M. A. (2008). Validez de contenido y juicio de expertos: una aproximación a su actualización. Avance en Medición, 6(1), 27-36.

Escurra, L. (1988). Cuantificación de la validez de contenido por criterio de jueces. Revista de Psicología, 6(1-2), 103-111.

Flamarique, I., Plana, M. T., Castro-Fornieles, J., Borràs, R., Moreno, E. \& Lázaro, L. (2019). Comparison of Perfectionism Dimensions in Adolescents with Anorexia Nervosa or Obsessive-Compulsive Disorder. Journal of the Canadian Academy of Child and Adolescent Psychiatry, 28(2), 45.

Flett, G. L. \& Hewitt, P. L. (2019). Reflections on Three Decades of Research on Multidimensional Perfectionism: An Introduction to the Special Issue on Further Advances in the Assessment of Perfectionism. Journal of Psychoeducational Assessment, 1-12. https://doi.org/10.1177/0734282919881928

Flett, G. L., Hewitt, P. L., Besser, A., Su, C., Vaillancourt, T., Boucher, D., Munro, Y., Davidson, L. A. \& Gale, O. (2016). The ChildAdolescent Perfectionism Scale: Development, psychometric properties, and associations with stress, distress, and psychiatric symptoms. Journal of Psychoeducational Assessment, 34(7), 634-652. https://doi.org/10.1177/0734282916651381

Flett, G. L., Hewitt, P. L., Boucher, D. J., Davidson, L. A. \& Munro, Y. (2000). The child-adolescent perfectionism scale: Development, validation, and association with adjustment. Unpublished manuscript. Recuperado de: https://hewittlab. psych.ubc.ca/measures-3/child-adolescent-perfectionismscale/
Foygel, R. \& Drton, M. (2010). Extended Bayesian information criteria for Gaussian graphical models. Advances in Neural Information Processing Systems, 23(1), 2020-2028.

Friedman, J., Hastie, T. \& Tibshirani, R. (2008). Sparse inverse covariance estimation with the graphical lasso. Biostatistics, 9(3), 432-441. https://doi.org/10.1093/biostatistics/kxm045

Frost, R. O., Heimberg, R. G., Holt, C. S., Mattia, J. I. \& Neubauer, A.L. (1993). A comparison of two measures of perfectionism. Personality and Individual Differences, 14(1), 119-126. https://doi.org/10.1016/0191-8869(93)90181-2

Frost, R. O., Marten, P., Lahart, C. \& Rosenblate, R. (1990). The dimensions of perfectionism. Cognitive Therapy and Research, 14(1), 449-468. https://doi.org/10.1007/bf01172967.

Fruchterman, T. \& Reingold, E. (1991). Graph drawing by forcedirected placement. Software: Practice and Experience, 21(1), 1129-1164.

https://doi.org/10.1002/spe.4380211102.

García-Fernández, J. M., Inglés, C. J., Vicent, M., Gonzálvez, C., Gómez-Núñez, M. I. y Poveda-Serra, P. (2016). Perfeccionismo durante la infancia y la adolescencia. Análisis bibliométrico y temático (2004-2014). Revista Iberoamericana de Psicología y Salud, 7(2), 79-88. https://doi.org/10.1016/j.rips.2016.02.001

Gavino, A., Nogueira, R., Pérez-Costillas, L. \& Godoy, A. (2017). Psychometric Properties of the Frost Multidimensional Perfectionism Scale in Spanish Children and Adolescents. Assessment, 26(3), 445464. https://doi.org/10.1177/1073191117740204

Hair, J.F., Anderson, R.E., Tatham, R.L., \& Black, W.C. (1995). Análisis Multivariante. (5a Ed.). Prentice Hall.

Hair, J.F., Black, W.C., Babin, B. J., \& Anderson, R.E. (2014). Multivariate Data Analysis (7a Ed.). Pearson.

Hewitt, P. L., Caelian, C. F., Flett, G. L., Sherry, S.B., Collins, L. \& Flynn, C. A. (2002). Perfectionism in children: Associations with depression, anxiety, and anger. Personality and Individual Differences, 32(6), 1049-1061. https://doi.org/10.1016/S01918869(01)00109-X

Hewitt, P. L. \& Flett, G. L. (1991). Perfectionism in the Self and Social Contexts: Conceptualization, Assessment, and Association with Psychopathology. Journal of Personality and Social Psychology. 60(3) 456-470. https://doi.org/10.1037/0022-3514.60.3.456

Hewitt, P. L., Flett, G. L., Turnbull-Donovan, W. \& Mikail, S. F. (1991). The Multi-dimensional Perfectionism Scale: Reliability, validity, and psychometric properties in psychiatric samples. Psychological Assessment, 3, 464-468. https://doi.org/10.1037/1040-3590.3.3.464

Hill, A. P. \& Curran, T. (2016). Multidimensional perfectionism and burnout: A meta-analysis. Personality and Social Psychology Review, 20(3),269-288.https://doi.org/10.1177/1088868315596286

Hofmann, S. G., Curtiss, J. \& McNally, R. J. (2016). A Complex Network Perspective on Clinical Science. Perspectives on Psychological Science, 11(5), 597-605. https://doi. org/10.1177/1745691616639283

Horn, J. L. (1965). A rationale and test for the number of factors in factor analysis. Psychometrika, 30(1), 179-185. https://doi. org/10.1007/bf02289447

Hu, L. T. \& Bentler, P. M. (1999). Cutoff criteria for fit indexes in covariance structure analysis: Conventional criteria versus new alternatives. Structural Equation Modeling, 6(1), 1-55. https://doi.org/10.1080/10705519909540118

JASP Team (2019). JASP (Version 0.11.) [Computer software]. 
Kaiser, H. F. (1970). A second-generation Little Jiffy. Psychometrika, 35(4), 401-415. https://doi.org/10.1007/BF02291817.

Kelly, A. C. \& Zuroff, D. C. (2013). Perfectionism. En L. Grossman y S. Valfish (Coords.), Translating psychological research into practice (pp. 233-236). Springer Publishing Company, LLC.

Klibert, J., Lamis, D. A., Naufel, K., Yancey, A.T. \& Lohr, S. (2015). Associations between perfectionism and generalized anxiety: Examining cognitive schemas and gender. Journal of Rational-Emotive Cognitive-Behavioral Therapy, 33(2), 160178. https://doi.org/10.1007/s10942-015-0208-9.

Kline, R. B. (2010). Principles and practice of structural equation modeling. Guilford Press.

Leone, E. M. \& Wade, T. (2017). Measuring perfectionism in children: a systematic review of the mental health literature. European Child and Adolescent Psychiatry, 27(1), 553-567. https://doi.org/10.1007/s00787-017-1078-8

Li, Y., Wen, Z., Hau, K. T., Yuan, K. H. \& Peng, Y. (2020). Effects of cross-loadings on determining the number of factors to retain. Structural Equation Modeling: A Multidisciplinary Journal, 1-23. https://doi.org/10.1080/10705511.2020.1745075

Limburg, K., Watson, H. J., Hagger, M. S. \& Egan, S. J. (2017). The relationship between perfectionism and psychopathology: A meta-analysis. Journal of Clinical Psychology, 73(10), 1301-1326. https://doi.org/10.1002/jclp.22435

Lloyd, S., Schmidt, U., Khondoker, M. \& Tchanturia, K. (2015). Can psychological interventions reduce perfectionism? A systematic review and meta-analysis. Behavioural and Cognitive Psychotherapy, 43(6), 705-731. https://doi. org/10.1017/S1352465814000162

Lorenzo-Seva, U. (1999). Promin: A method for oblique factor rotation. Multivariate Behavioral Research, 34(3), 347-365. https://doi.org/10.1207/S15327906MBR3403_3

Mardia, K. V. (1970). Measures of multivariate skewness and kurtosis with applications. Biometrika, 57(3), 519-530. https://doi.org/10.2307/2334770

McNeish, D. \& Wolf, M. G. (2020). Thinking twice about sum scores. Behavior Research Methods. https://doi.org/10.3758/ s13428-020-01398-0

Morris, L. \& Lomax, C. (2014). Review: Assessment, development, and treatment of childhood perfectionism: a systematic review. Child and Adolescent Mental Health, 19(4), 225-234. https:// doi.org/10.1111/camh.12067

Muñiz, J., Elosua, P. \& Hambleton, R. (2013). Directrices para la traducción y adaptación de los tests: segunda edición. Psicothema, 25(2), 151-157. HTTPS://DOI.ORG/ 10.7334/psicothema2013.24.

Muthén, B. \& Kaplan, D. (1985). A comparison of some methodologies for the factor analysis of non-normal Likert variables. British Journal of Mathematical and Statistical Psychology, 38(2), 171189. https://doi.org/10.1111/j.20448317.1985.tb00832.x.

Nigar, A. \& Naqvi, I. (2019). Body dissatisfaction, perfectionism, and media exposure among adolescents. Pakistan Journal of Psychological Research, 34(1), 57-77. https://doi. org/10.33824/pjpr.2019.34.1.4

Núñez, C., Ramos-Vera, C.A., Serpa, A. y Ogundokun, R.O. (2021). Adaptation of the Personality Type Inventory Based on Enneagram in Peruvian university students of health sciences. Journal of Research in Medical and Dental Science, 9(5). doi:10.13140/RG.2.2.33090.30404
Opsahl, T., Agneessens, F. \& Skvoretz, J. (2010). Node centrality in weighted networks: Generalizing degree and shortest paths. Social Networks, 32(3), 245-251. https://doi.org/10.1016/j. socnet.2010.03.006

Oros, L. B. (2003) Medición del perfeccionismo infantil: desarrollo y validación de una escala para niños de 8 a 13 años de edad. Revista iberoamericana de diagnóstico y evaluación psicológica, 16(2), 99-112.

Oros, L.B., Iuorno, O. \& Serppe, M. (2017). Child perfectionism and its relationship with personality, excessive parental demands, depressive symptoms and experience of positive emotions. The Spanish Journal of Psychology, 20.e9, 1-13. https://doi.org/10.1017/sjp.2017.9

Ramos Vera, C. A. (2020). Un método de cálculo del tamaño muestral en modelos de ecuaciones estructurales. Revista Perspectiva Empresarial, 7(2), 7-9. doi:10.16967/23898186.659

Ramos-Vera, C. (2021a). Las redes de relación estadística en la investigación psiquiátrica: el caso del delirio en el contexto de COVID-19. Revista Colombiana De Psiquiatria. doi: 10.1016/j. rcp.2021.02.004

Ramos-Vera, C.A. (2021b). Las redes de relación estadística en la investigación de nutrición. Nutrición Hospitalaria. DOI: 10.20960/nh.03522

Ramos-Vera, C. A. (2021c). Conceptos de interés en la atención integral de los pacientes con multimorbilidad. Atencion Primaria, 53(6).101969. doi: 10.1016/j.aprim.2021.101969

Ramos-Vera, C., Baños-Chaparro, J. \& Ogundokun R. O. (2021). Network structure of depressive symptoms in Peruvian adults with arterial hypertension. F1000Research, 10,19. doi: 10.12688/ f1000research.27422.2

Ramos-Vera, C., y Motta. J. C. (2021). Factores pronósticos en pacientes hospitalizados con diagnóstico de infección por SARSCoV-2 en Bogotá, Colombia. Biomédica, 41(2), 374-377. https:// revistabiomedica.org/index.php/biomedica/article/view/6206

Ramos-Vera, C. y Serpa, A. (2021). Network analysis in psychiatric research. Actas Españolas de Psiquiatría, 49(3), 123-124.

Reynolds, W. M. (1987). Reynolds Adolescent Depression Scale. Professional manual. Psychological Assessment Resources, Inc.

Rimm, S. (2007). What's wrong with perfect? clinical perspectives on perfectionism and underachievement. Gifted Education International, 23(3), 246-253. https://doi.org/10.1177/ 026142940702300305

Satorra, A. \& Bentler, P. M. (2001). A scaled difference chi-square test statistic for moment structure analysis. Psychometrika, 66(4), 507-514. https://doi.org/10.1007/bf02296192

Sherry, S. B., Mackinnon, S. P. \& Nealis, L. J. (2018). Perfectionism and Interpersonal Problems: Narcissistic and Self-Critical Perfectionism. In J. Stoeber(Ed.), The Psychology of Perfectionism: Theory, Research, Applications (pp. 177-200). Routledge.

Sironic, A. \& Reeve, R.A. (2015). A combined analysis of the Frost Multidimensional Perfectionism Scale (FMPS), Child and Adolescent Perfectionism Scale (CAPS), and Almost Perfect Scale-Revised (APSR): Different perfectionist profiles in adolescent high school students. Psychological Assessment, 27(1), 1471-1483. https://doi.org/10.1037/pas0000137

Slade, P. D. \& Owens, R. G. (1998). A dual process model of perfectionism based on reinforcement theory. Behavior modification, 22(3), 372-390. https://doi.org/10.1177/ 01454455980223010 
Smith, M. M., Saklofske, D. H., Stoeber, J. \& Sherry, S.B. (2016). The big three perfectionism scale: A new measure of perfectionism. Journal of Psychoeducational Assessment, 34(7), 670-687. https://doi.org/10.1177/0734282916651539

Starley, D. (2018). Perfectionism: a challenging but worthwhile research area for educational psychology. Educational Psychology in Practice, 35(2), 1-26. https://doi.org/10.1080/0 2667363.2018.1539949

Stoeber, J. (2018). The psychology of perfectionism: An introduction. In J. Stoeber (Ed.), The psychology of perfectionism: Theory, Research, Applications (pp. 3-16). Routledge

Stoeber, J. \& Childs, J. H. (2012). Perfectionism. In Levesque R. J. R. (Ed.), Encyclopaedia of adolescence (pp. 2053-2059). Springer.

Stoeber, J. \& Gaudreau, P. (2017). The advantages of partialling perfectionistic strivings and perfectionistic concerns: Critical issues and recommendations. Personality and Individual Differences, 104(1), 379-386. https://doi.org/10.1016/j.paid.2016.08.039

Stoeber, J. \& Otto, K. (2006). Positive Conceptions of Perfectionism: Approaches, Evidence, Challenges. Personality and Social Psychology Review 10(4) 295-319. https://doi.org/10.1207/ s15327957pspr1004_2

Ugarriza, N. y Escurra, M. (2002). Adaptación psicométrica de la Escala de Depresión para Adolescentes de Reynolds (EDAR) en estudiantes de secundaria de Lima Metropolitana. Persona, 5(1), 83-130. https://doi.org/10.26439/persona2002.n005.872
Van de Vijver. F. J. R. \& Hambleton, R. K. (1996). Translating tests: Some practical guidelines. European Psychologist, 1, 89-99. https://doi.org/10.1027/1016-9040.1.2.89

Van der Kaap-Deeder, J., Smets, J. \& Boone, L. (2016). The Impeding Role of Self-Critical Perfectionism on Therapeutic Alliance During Treatment and Eating Disorder Symptoms at Follow-up in Patients with an Eating Disorder. Psychologica Belgica, 56(2), 101-110. http://dx.doi.org/10.5334/pb.297

Ventura, J., Jara, S., García, C. y Ortiz, C. (2018). Validación de una escala de perfeccionismo en niños peruanos. Actualidades en Psicología, 32(124), 15-32. https://doi.org/10.15517/ap.v32i124.30385.

Vicent, M., Inglés, C. J., Sanmartín, R., Gonzálvez, C., Delgado, B. \& García-Fernández, J. M. (2019). Spanish Validation of the Child and Adolescent Perfectionism Scale: Factorial Invariance and Latent Means Differences across Sex and Age. Brain Sciences, 9(11), 310. https://doi.org/10.3390/brainsci9110310

Vicent, M., Inglés, C. J., Sanmartín, R., Gonzálvez, C., JiménezAyala, C.E. \& García-Fernández, J. M. (2020). Psychometric Properties of the Child and Adolescent Perfectionism Scale in Ecuadorian Adolescents. Journal of Affective Disorders. 272, 176-182. https://doi.org/10.1016/j.jad.2020.04.036

Xu, M. L. \& Leung, S. O. (2018). Effects of varying numbers of Likert scale points on factor structure of the Rosenberg SelfEsteem Scale. Asian Journal of Social Psychology, 21(3), 119-128. https://doi.org/10.1111/ajsp.12214 